\title{
Uniformly Dispersed Carbide Reinforcements in the Medium-Entropy High-Speed Steel Coatings by Wide-Band Laser Cladding
}

\author{
Bang Dou ${ }^{1,2} \cdot$ Hui Zhang ${ }^{1,2} \cdot$ Jia-Hao Zhu ${ }^{1} \cdot$ Ben-Qi Xu $\cdot$ Zi-Yi Zhou ${ }^{3} \cdot \mathrm{Ji}^{1}-\mathrm{Li} \mathrm{Wu}^{3}$
}

Received: 4 September 2019 / Revised: 15 November 2019 / Published online: 6 January 2020

(c) The Chinese Society for Metals (CSM) and Springer-Verlag GmbH Germany, part of Springer Nature 2020

\begin{abstract}
A medium-entropy high-speed steel (ME-HSS) coating with the 76 at.\% of $\mathrm{Fe}$ and multiple alloying elements was prepared by the wide-band laser cladding. Compared with the commercial $\mathrm{W}_{6} \mathrm{Mo}_{5} \mathrm{Cr}_{4} \mathrm{~V}_{2}$ (M2) HSS coating which contains a large number of network lamellar $M_{2} \mathrm{C}$-type carbides along the grain boundaries, the presented ME-HSS coating has a high quantity of finer and more uniformly dispersed $M C$-type carbides; on the other hand, the coating has less retained austenite and much lower brittleness as well as similar secondary hardening effect and tempering hardness.
\end{abstract}

Keywords High-speed steel $\cdot$ Medium-entropy alloy $\cdot$ Phase selection $\cdot$ Laser cladding

\section{Introduction}

The so-called medium-entropy alloys (MEAs) were provoked by the research boom in the high-entropy alloys (HEAs) and have been as a hot topic in the novel metallic materials. One-component designing strategy for MEAs is to prepare ternary or quaternary alloys with nearly equiatomic compositions. $\mathrm{CrCoNi}$ and $\mathrm{CrCoFeNi}$ MEAs are the well-known components and have been demonstrated to be of high strength, excellent ductility and cryogenic fracture resistance via the nano-twinning strengthening [1-3]. Another strategy is to improve the additive content of a single element exceeding 35 at.\% in the as-designed multicomponent alloys with the configuration entropy between $\mathrm{R}$ and

Available online at http://link.springer.com/journal/40195

Hui Zhang

huizhang@ahut.edu.cn

Ji-Li Wu

wujili@msn.com

1 Key Laboratory of Green Fabrication and Surface Technology of Advanced Metal Materials (Ministry of Education), Anhui University of Technology, Ma' anshan 243002, China

2 School of Materials Science and Engineering, Anhui University of Technology, Ma' anshan 243002, China

3 School of Materials Science and Engineering, Jiangsu University, Zhenjiang 212013, China
1.5R [4-10]. Jose et al. [6] and Choi et al. [7] separately reported the $\mathrm{Fe}_{50} \mathrm{Mn}_{30} \mathrm{Co}_{10} \mathrm{Cr}_{10}$ and $\mathrm{Fe}_{50}(\mathrm{CoCrMnNi})_{50}$ alloys with excellent strength and ductility by a conventional arc-melting technique. Zhang et al. prepared a nonequiatomic $\mathrm{Fe}_{6} \mathrm{NiCoCrAlTiSi}$ coating by laser cladding and proved that equimolarity was not a precondition for obtaining a single-phase solid solution in the HEAs [8]. Yao et al. [9] and Zhuang et al. [10] also, respectively, prepared the non-equiatomic $\mathrm{Fe}_{40} \mathrm{Co}_{5} \mathrm{Cr}_{2} \mathrm{Mn}_{27} \mathrm{Ni}_{26}$ and $\mathrm{FeCo}_{3} \mathrm{NiCuAl}$ alloys with simple solid-solution phases by a suck-casting method. Therefore, the concept of Fe-rich MEAs, which contains more than 50 at.\% of $\mathrm{Fe}$, is expected to greatly reduce the consumption of expensive elements like $\mathrm{Co}$ and $\mathrm{Ni}$ in the HEAs and thus to extend the potential application in industrial community.

In our previous work, a medium-entropy (ME) highspeed steel (HSS) coating with Fe content of 68 at.\% by laser cladding was synthesized [11]. The coating possessed excellent oxidative hot wear resistance than the M2-HSS $\left(\mathrm{W}_{6} \mathrm{Mo}_{5} \mathrm{Cr}_{4} \mathrm{~V}_{2}\right)$ coating, because the former contains a high content of anti-oxidation elements, such as $\mathrm{Cr}$, $\mathrm{Co}$ and $\mathrm{Al}$, and hence leads to a more continuous and compact oxidation film on the worn surface after hot wear tests at $500{ }^{\circ} \mathrm{C}$. Therefore, the ME-HSS component can be expected to partially replace the conventional HSS coating serving as iron-based thermal wear-resistant material, such as on the surface of hot working roll. However, industry application always pursues to reduce the cost and improves processing efficiency. Therefore, the current work aims to improve the 
iron content of ME-HSS coating from 68 to 76 at.\% with the addition of more alloying elements like $\mathrm{Cu}$ and $\mathrm{Ti}$, based on the previous works $[11,12]$. The components of the newly proposed ME-HSS coating and a widely industrial used M2-HSS $\left(\mathrm{W}_{6} \mathrm{Mo}_{5} \mathrm{Cr}_{4} \mathrm{~V}_{2}\right)$ coating are comparably presented in Table 1. The weight percentage of $\mathrm{W}, \mathrm{Mo}$ and $\mathrm{C}$ is controlled to be equal in the two alloys. Other elements are set to be 2.1 at. \% in the ME-HSS component in order to improve the content of the alloying elements and lead to enhance the entropic effect. Furthermore, the wide-band laser cladding technique with spot size $15 \mathrm{~mm} \times 2 \mathrm{~mm}$ was utilized to accelerate the coating forming efficiency, in comparison with laser spot of $4 \mathrm{~mm}$ diameters in our previous works $[11,12]$.

\section{Experimental}

The ME-HSS powder was prepared by mechanically mixing the M2-HSS powder (Höganäs Company) with other required elemental powders according to the composition listed in Table 1. The motivation of choosing M2-HSS powder as a raw material was to improve the coating cladding properties, because the purchased powder prepared by gas atomization has a more suitable sphericity and particle size for laser cladding. A layer of ME-HSS powder bed with about $1.0 \mathrm{~mm}$ thickness was preset onto a steel substrate and cladded by the LDM 4000-100 diode laser. The specific laser parameters were $3 \mathrm{~kW}$ of power, $15 \mathrm{~mm} \times 2 \mathrm{~mm}$ of rectangular beam spot and $300 \mathrm{~mm} \mathrm{~min}^{-1}$ of scanning speed. Argon flux was used to prevent the oxidation during the cladding process. Furthermore, a comparative M2-HSS coating was prepared under the same process. After the laser cladding, the coating was tempered three times with each hold time of $20 \mathrm{~min}$ in the $470-590{ }^{\circ} \mathrm{C}$ temperature range. The purpose of the triple tempering treatment was to strengthen the matrix by secondary hardening and eliminate the possibly existed retained austenite [13, 14].

A Rigaku X-ray diffractometer (XRD) $(\mathrm{Cu} K \alpha)$ was used to identify the phase structure in the coating. The microstructure was characterized by a field emission scanning electron microscope (SEM, Quanta 450). The elemental composition and distribution were determined by an energy-dispersive spectrometer (EDS) equipped with SEM. The Vickers hardness of the coatings was measured on a standard HMV-2T
Vickers hardness tester. The test pressure and dwell time were fixed at $4.9 \mathrm{~N}$ and $20 \mathrm{~s}$, respectively, and each sample was measured nine times to calculate the average hardness. The brittleness of the coatings was evaluated via the length of cracks provoked by Vickers indentation at the corners of Vickers pyramid under a large loading force of $294 \mathrm{~N}$.

\section{Results and Discussion}

\subsection{Coating Morphology and Structure}

Figure 1a shows the smooth surface morphology with the width of ca. $15 \mathrm{~mm}$ in the single-track ME-HSS coating. The XRD patterns in Fig. $1 \mathrm{~b}$ show that the coatings are mainly composed of martensite matrix, retained austenite and $M C$ type and $M_{2} \mathrm{C}$-type reinforcements after laser cladding. After the triple-tempering at $530{ }^{\circ} \mathrm{C}$, the residual austenite completely disappears and transforms to martensite. This microstructure transition is almost same as most of the previous results on the traditional $\mathrm{Fe}-\mathrm{C}-\mathrm{X}$ HSS coating prepared by laser cladding [15]. The main difference in the controllable coatings is that the dominant carbide in the M2-HSS coating is $M_{2} \mathrm{C}$ type, but the carbide transforms to $M C$ in the $\mathrm{ME}$ HSS coating. Therefore, it is convinced that the proposed medium-entropy alloy coating is reasonably classified to the HSS alloy system.

\subsection{Microstructure and Hardness Evolution}

Figure 2 shows the cross-sectional microstructure at the center of the two coatings. The microstructure of solidified M2-HSS coating (Fig. 2a) is characterized by an acicular martensitic matrix separated by the network mixture of the retained austenite and carbides. After the triple tempering at $530{ }^{\circ} \mathrm{C}$, Fig. $2 \mathrm{~b}$ shows that the retained austenite almost disappears and a high quantity of lamellar carbides can be observed at grain boundaries. The carbide network has the typical lamellar morphology as $M_{2} \mathrm{C}$-type carbide, which has been reported in the previous works $[16,17]$.

Figure $2 \mathrm{c}$ shows that the content of network mixture of retained austenite and carbides is much less in the ME-HSSsolidified coating than those in the M2-HSS coating. Furthermore, Fig. $2 \mathrm{~d}$ shows that more carbides are uniformly dispersed in the martensitic matrix after the tempering
Table 1 Compositions and configuration entropies of the laser-cladded ME-HSS coatings

\begin{tabular}{llllllllllllll}
\hline & $\%$ & $\mathrm{C}$ & $\mathrm{W}$ & $\mathrm{Mo}$ & $\mathrm{Cr}$ & $\mathrm{V}$ & $\mathrm{Co}$ & $\mathrm{Ni}$ & $\mathrm{Al}$ & $\mathrm{Cu}$ & $\mathrm{Ti}$ & $\mathrm{Fe}$ & Entropy \\
\hline ME-HSS & at. & 4.5 & 1.85 & 2.95 & 2.1 & 2.1 & 2.1 & 2.1 & 2.1 & 2.1 & 2.1 & 76 & $1.09 \mathrm{R}$ \\
& wt. & 0.95 & 6.0 & 5.0 & 1.92 & 1.88 & 2.18 & 2.18 & 1.0 & 2.35 & 1.77 & 74.78 & \\
M2-HSS & at. & 4.5 & 1.8 & 2.9 & 4.4 & 2.2 & - & - & - & - & - & 83.9 & $0.69 \mathrm{R}$ \\
& wt. & 0.95 & 6.0 & 5.0 & 4.0 & 2.0 & - & - & - & - & - & 82.05 & \\
\hline
\end{tabular}



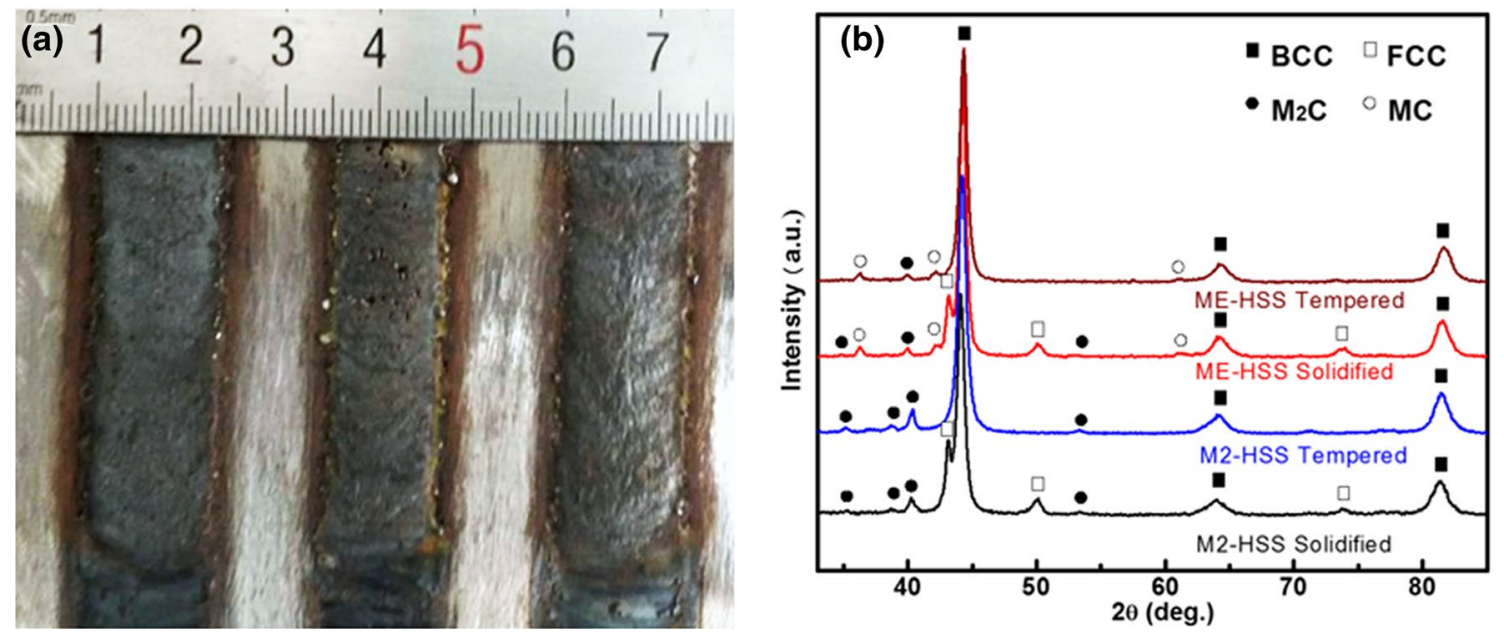

Fig. 1 a Smooth surface on the ME-HSS coating, b XRD patterns of the two coatings in the solidified state and after triple tempering at $530{ }^{\circ} \mathrm{C}$
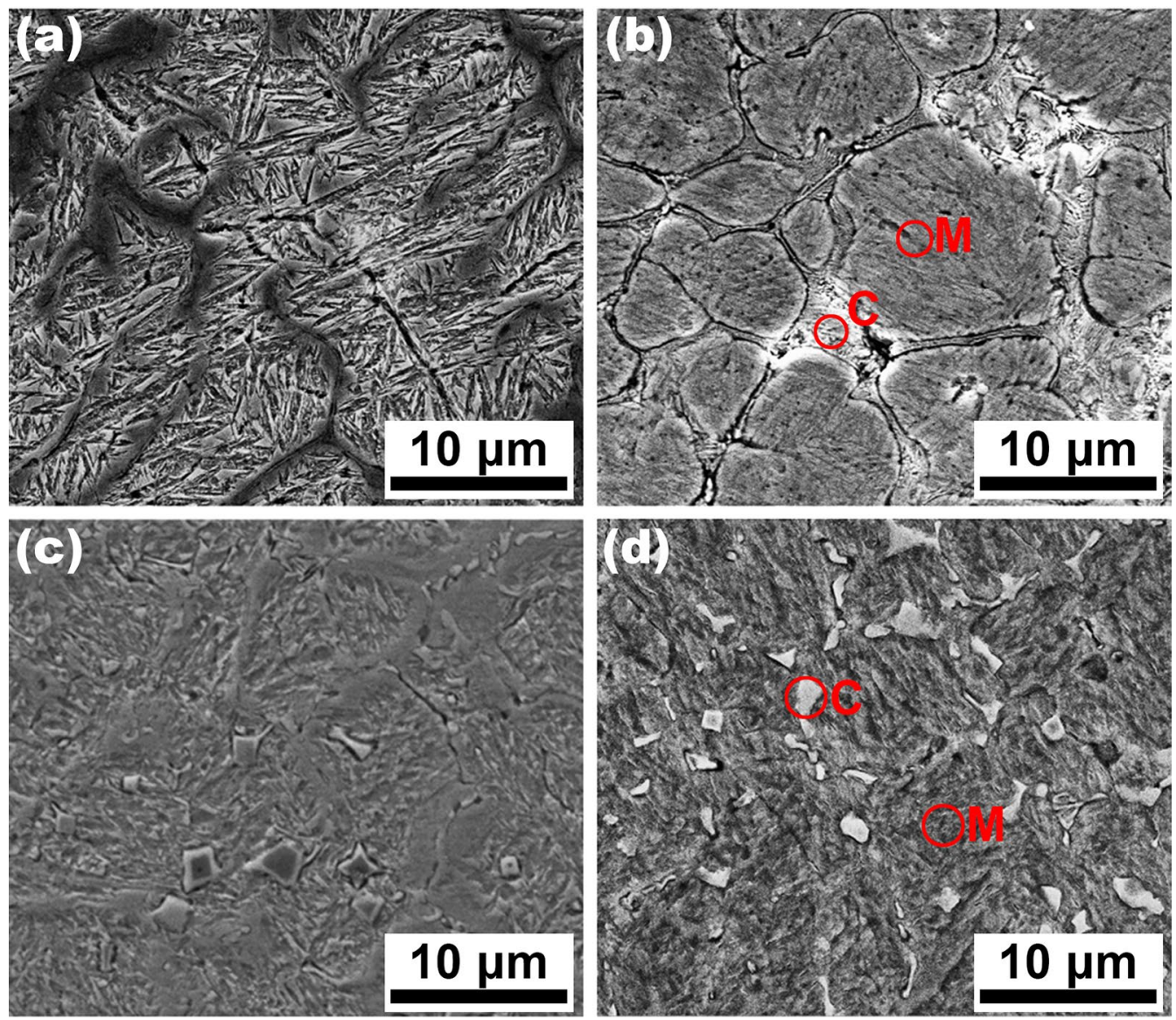

Fig. 2 Microstructure of the two coatings in the solidified state and after triple tempering with the label $\mathrm{C}$ for carbides, and $\mathrm{M}$ for martensite: a, b M2-HSS coating; c, d ME-HSS coating

treatment. The network carbide distribution is broken and almost completely disappeared. EDS analysis compares the chemical composition in the carbides (labeled as C) with the martensitic matrix (labeled as $\mathbf{M}$ ) after the tempering treatment. It is found that the carbides are mainly enriched in $\mathrm{W}, \mathrm{Mo}, \mathrm{Cr}$ and $\mathrm{V}$ elements in the M2-HSS coating, while the main difference is that Ti greatly is involved in carbide phases in the ME-HSS coating. 
Figure 3 shows the interface microstructure between the coating and substrate and the component distribution of main alloying elements along the EDS scanning line. The purple, green, red and blue lines refer to iron, molybdenum, chromium and tungsten elements, respectively. Component scanning lines of other additive elements, such as $\mathrm{Al}$ and $\mathrm{Ni}$, were not presented here avoiding difficulty to be distinguished. The results indicate that the carbides are also well distributed near the interface of the coating. There is a clear interface for the elemental distribution between the coating and substrate. The component transition region, where the $\mathrm{Cr}, \mathrm{W}$ and Mo alloying elements gradually increased from the interface to the coating, is very narrow with a thickness of about $5 \mu \mathrm{m}$, suggesting that the component dilution is very low in the ME-HSS coating.

Figure 4a shows the obvious secondary hardening both occurred in the compared coatings after triple tempering in the temperature range of $470-590{ }^{\circ} \mathrm{C}$. The maximum tempering hardness in the ME-HSS and M2-HSS coatings reaches about $827 \mathrm{HV}_{0.5}$ and $840 \mathrm{HV}_{0.5}$, respectively, which both exceed the hardness of about $800 \mathrm{HV}$ in the traditional casting prepared $\mathrm{Fe}-\mathrm{C}-\mathrm{X}$ HSS $[14,18,19]$. Figure $4 \mathrm{~b}$ and $\mathrm{c}$ shows the comparison of the indentation brittleness of the two coatings in the highest tempering hardness state by the Vickers indentation method. This method can evaluate the brittleness for high-brittle and high-hardness materials (poor toughness and resistance to propagation) by the growth length of a crack initiated at the corners of the Vickers pyramid [20]. The M2-HSS coating has much higher brittleness with the cracks obviously observed at the corner of the indentation. Therefore, it is believed that the fine and

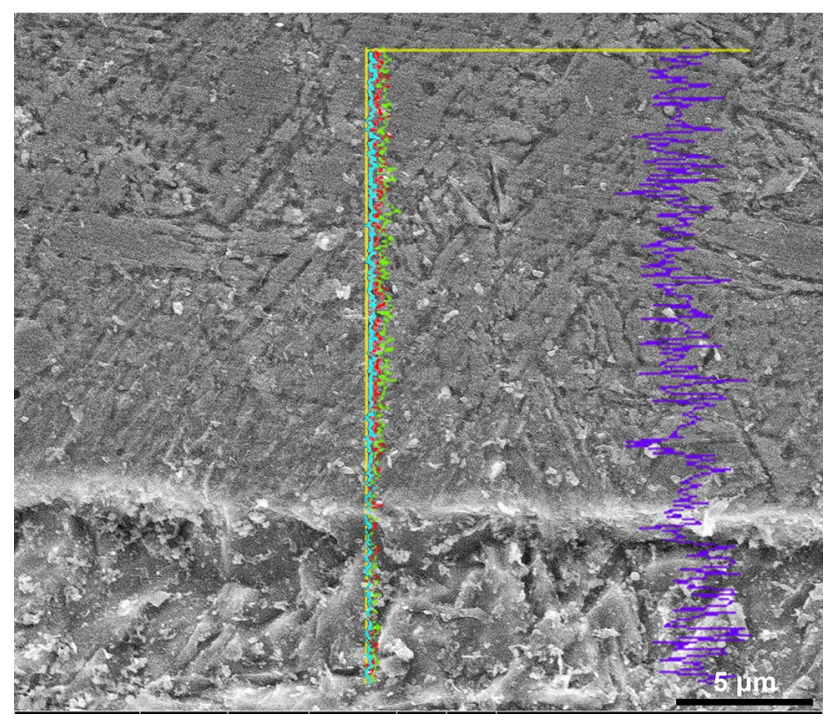

Fig. 3 Interface microstructure between the coating and substrate with EDS scanning lines (the purple, green, red, and blue lines refer to iron, molybdenum, chromium, and tungsten elements, respectively) well-dispersed carbides bring lower brittleness in the MEHSS coating.

\subsection{Formation Reasons of Uniform Carbide Dispersion in the ME-HSS Coating}

The hardness of the HSS mainly depends on the type of the carbides, in which the higher hardness and the finer and more uniformly distributed microstructure are much pursued $[14,21,22]$. It is already known that the hard $M C$ carbides are proeutectoid phase and can be uniformly dispersed inside grains [21], while other carbides like $M_{2} \mathrm{C}, M_{6} \mathrm{C}$ and $M_{7} \mathrm{C}_{3}$ come from the eutectic reaction and mainly distributed at the grain boundaries $[14,16]$. The reported Vickers hardness of the different carbides was $M C$ (2100-2800 HV), $M_{2} \mathrm{C}(1800-2250 \mathrm{HV}), M_{6} \mathrm{C}(1800-2250 \mathrm{HV})$ and $M_{7} \mathrm{C}_{3}$ (1890-2060 HV) [22]. Therefore, $M C$ is the most favorably hardest carbide and can be uniformly distributed. However, the obtained carbides are more likely to be $M_{2} \mathrm{C}$ and $M_{6} \mathrm{C}$ in the traditional casting prepared $\mathrm{Fe}-\mathrm{C}-\mathrm{X}$ HSS [21-23]. It is rarely reported that $M C$ can be obtained as main carbide reinforcement and the network carbide formation can be completely avoided.

In this work, uniformly dispersed $M_{2} \mathrm{C}$ and $M C$ carbides were obtained in the ME-HSS coating. The high content of $M C$ obtained in the ME-HSS coating should be attributed to the medium-entropy effect which suppresses the nucleation of the carbides causing a lower content of the carbides in the ME-HSS coating than that in the M2-HSS coating. According to the mixing enthalpy in the different types of carbides, $M C$ carbide is firstly precipitated from the liquid and more stable than other carbides in the HSS system [14, 17]. Therefore, the inhibition for $M C$ nucleation is the relatively weak and $M C$ becomes the dominant reinforcement in the ME-HSS coating. Furthermore, it is known that TiC has strong formation free energy [24]. As a consequence, the additive element of $\mathrm{Ti}$ is another reason to promote the $M \mathrm{C}$ nucleation in the ME-HSS coating. This analysis is primarily supported by a high Ti content in the $M C$ carbide, according to EDS results (Table 2). Alternatively, adding Ti alloying element is a main difference between the current work and our previous works $[11,12]$. High-quantity and uniformly distributed $M C$ carbides were not clearly observed previously, suggesting that $\mathrm{Ti}$ is responsible for the formation of a high quantity of $M C$ in this work.

It is well known that solidification rate strongly affects the carbide distribution in the HSS coatings [14, 25]. Figure 5 displays the microstructure evolution in the MEHSS coatings with thicker powder bed prepared under the same laser processing parameters. It is demonstrated that a thinner powder bed thickness generally refers to a higher solidification rate under the same laser energy input [26]. Figure 5a validates that the networks of carbide phase 

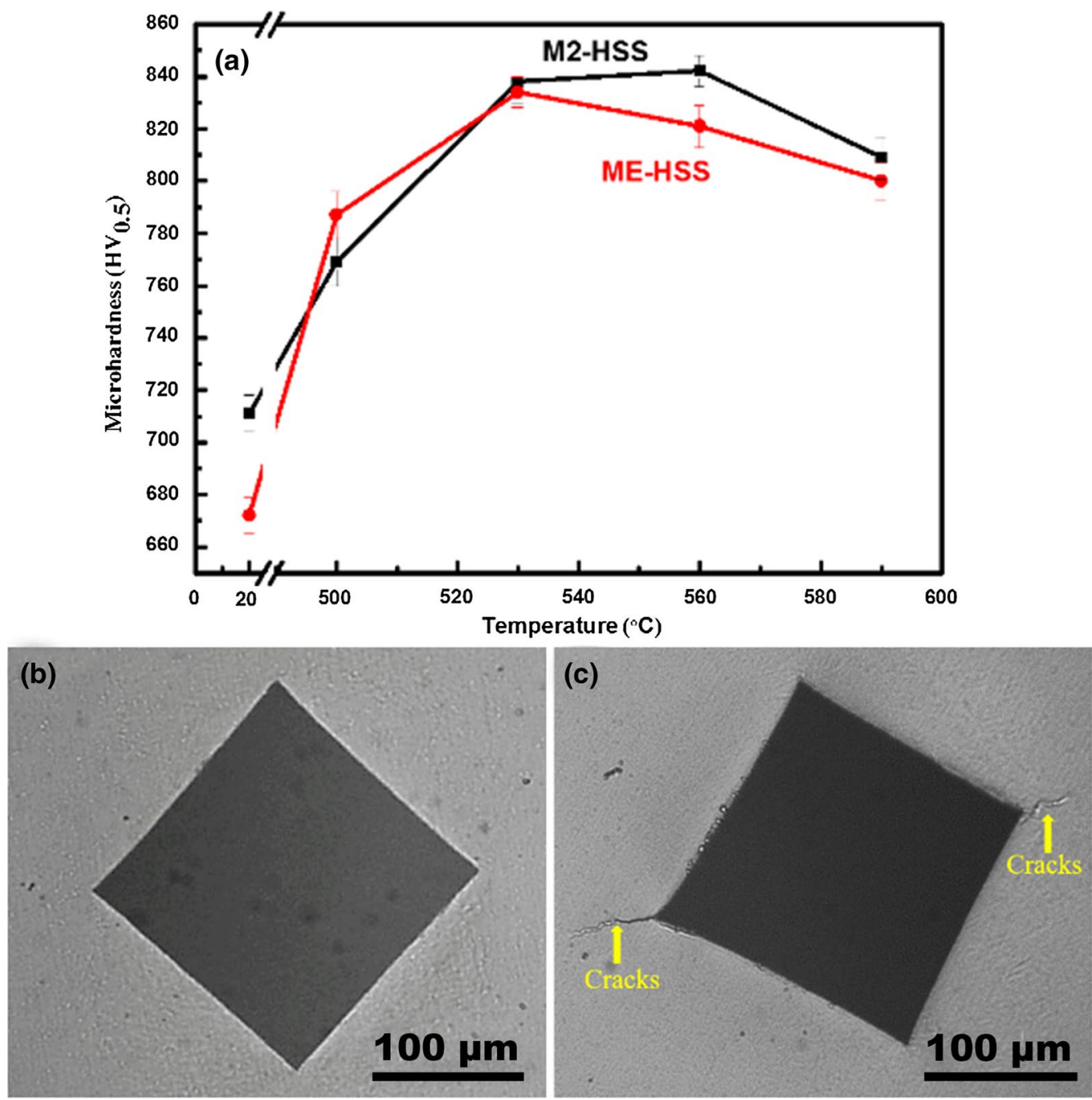

Fig. 4 a Hardness variation as a function of tempering temperature, b, c cracks observation at the indentations in the M2-HSS and ME-HSS coatings, respectively

Table 2 EDS results in various regions (Fig. 2) after triple tempering at $530{ }^{\circ} \mathrm{C}(\mathrm{at} \%)$

\begin{tabular}{lllrrrlllllll}
\hline Coatings & Regions & $\mathrm{C}$ & \multicolumn{1}{c}{$\mathrm{Mo}$} & $\mathrm{W}$ & $\mathrm{Cr}$ & $\mathrm{V}$ & $\mathrm{Co}$ & $\mathrm{Ni}$ & $\mathrm{Al}$ & $\mathrm{Cu}$ & $\mathrm{Ti}$ & $\mathrm{Fe}$ \\
\hline \multirow{2}{*}{ M2-HSS } & Carbide & 4.15 & 15.37 & 8.11 & 10.04 & 8.98 & & - & & - & - & 53.35 \\
& Matrix & 1.41 & 2.75 & 1.89 & 4.35 & 1.72 & & - & & - & - & 87.30 \\
\multirow{2}{*}{ ME-HSS } & Carbide & 7.75 & 13.16 & 10.44 & 2.35 & 10.43 & 0.16 & 0.37 & 0.13 & 2.12 & 14.88 & 38.21 \\
& Matrix & 2.54 & 1.26 & 0.89 & 1.87 & 1.75 & 3.48 & 3.47 & 4.47 & 3.46 & 0.56 & 76.25 \\
\hline
\end{tabular}

are still existed in the thick coatings with powder bed of $2 \mathrm{~mm}$. Figure $5 \mathrm{~b}$ shows the network is gradually broken up in the coating with powder bed of ca. $1.5 \mathrm{~mm}$, but the uniformity of carbide distribution is not as good as that of in the 1-mm-thick coating, as compared with Fig. 2d. The circles marked $M C$ carbides are still mainly distributed at the grain boundaries (Fig. 5b). This phenomenon indicates that the higher solidification rate favors the more uniform distribution of carbide in the ME-HSS coating. Because rapid solidification causes a high quantity of alloying elements to accumulate at the front of the solid-liquid interface, the enrichment of $\mathrm{Mo}, \mathrm{Co}, \mathrm{Ni}$ and $\mathrm{Al}$ in the residual liquid is all believed to promote the formation of $M_{2} \mathrm{C}$ (instead, W is beneficial to the formation of $M_{6} \mathrm{C}$ and $\mathrm{V}$ facilitate the formation of $M C$ ) $[14,27]$. Therefore, a fast solidification rate is required for the preparation of the ME-HSS coating. The thickness of about $1 \mathrm{~mm}$ with uniform carbide dispersion obtained in the current work can satisfy the industrial requirements for the wear-resistant coating thickness. 

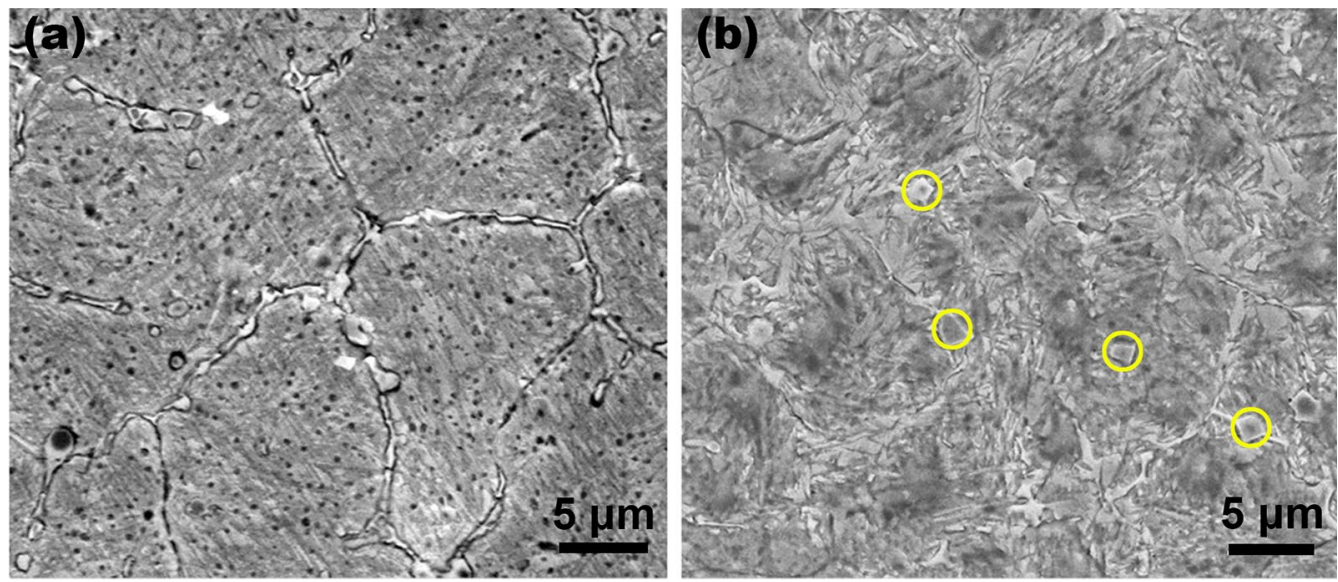

Fig. 5 Microstructure in the ME-HSS coatings with thicker powder bed: a $2 \mathrm{~mm}$ thickness; $\mathbf{b} 1.5 \mathrm{~mm}$ thickness

\section{Conclusions}

1. Uniformly dispersed $M \mathrm{C}$ and $M_{2} \mathrm{C}$ carbides with the martensitic matrix are obtained in a newly designed ME-HSS coating by wide-band laser cladding.

2. The ME-HSS coating has lower brittleness and similar maximum tempering hardness of about $827 \mathrm{HV}$, compared with the M2-HSS coating prepared under the same condition.

3. The formation reasons of uniform carbide dispersion in the ME-HSS coating are attributed to rapid solidification and a high content of alloying elements, particularly Ti.

Acknowledgements This work is financially supported by the National Natural Science Foundation of China (Nos. 51971001, U1560105 and 51601050) and the Open Fund from State Key Laboratory of Solid Lubricating (No. LSL-1714).

\section{References}

[1] H. Yang, X.L. Shang, L.L. Wang, Z. Wang, J.C. Wang, X. Lin, Acta Metall. Sin. 54, 905 (2018)

[2] B. Gludovatz, A. Hohenwarter, K.V.S. Thurston, H. Bei, Z. Wu, E.P. George, R.O. Ritchie, Nat. Commun. 7, 10602 (2016)

[3] H. Feng, H.B. Li, P.C. Lu, C.T. Yang, Z.H. Jiang, X.L. Wu, Acta Metall. Sin. 55, 1457 (2019)

[4] H. Zhang, H. Tang, W.H. Li, J.L. Wu, X.C. Zhong, Mater. Sci. Technol.-Lond. 34, 1 (2017)

[5] F. He, Z. Wang, M. Zhu, J. Li, Y. Dang, J. Wang, Mater. Des. 85, 1 (2015)

[6] Y.A. Jose, V.U. Alejandro, Z.M. Dario, P.H. Rodrigo, Mater. Sci. Eng. A 748, 244 (2019)

[7] M. Choi, I. Ondicho, N. Park, N. Tsuji, J. Alloys Compd. 780, 959 (2019)
[8] H. Zhang, Y. Pan, Y. He, H. Jiao, Appl. Surf. Sci. 257, 2259 (2011)

[9] M.J. Yao, K.G. Pradeep, C.C. Tasan, D. Raabe, Scr. Mater. 72-73, 5 (2014)

[10] Y.X. Zhuang, W.J. Liu, P.F. Xing, F. Wang, J.C. He, Acta Metall. Sin. (Engl. Lett.) 25, 124 (2012)

[11] H. Tang, H. Zhang, L. Chen, S. Guo, J. Alloys Compd. 772, 719 (2019)

[12] H. Zhang, B. Dou, H. Tang, Y.Z. He, S. Guo, Mater. Des. 159, 224 (2018)

[13] X.U. Bojun, G.U. Nanju, Y. Dianran, Acta Metall. Sin. (Engl. Lett.) 3, 116 (1990)

[14] M. Boccalini, H. Goldenstein, Int. Mater. Rev. 46, 92 (2001)

[15] W. Darmawan, J. Quesada, F. Rossi, R. Marchal, F. Machi, H. Usuki, J. Laser Appl. 21, 176 (2009)

[16] X. Zhou, D. Liu, W. Zhu, F. Fang, Y. Tu, J. Jiang, J. Iron. Steel Res. Int. 24, 43 (2017)

[17] F. Pan, W. Wang, A. Tang, L. Wu, T. Liu, R. Cheng, Prog. Nat. Sci. Mater. 21, 180 (2011)

[18] S. Wei, J. Zhu, L. Xu, Mater. Sci. Eng. A 404, 138 (2005)

[19] V. Trabadelo, S. Giménez, I. Iturriza, Mater. Sci. Eng. A 499, 360 (2009)

[20] V. Keryvin, V.H. Hoang, J. Shen, Intermetallics 17, 211 (2009)

[21] G. Herranz, A. Romero, V. de Castro, G.P. Rodríguez, Mater. Des. 54, 934 (2014)

[22] H.W. Zhang, K. Nakajima, M. Su, H. Shibata, P. Hedström, W. Wang, H. Lei, Q. Wang, P.G. Jönsson, J. He, Steel Res. Int. 89, 1800172 (2018)

[23] X.F. Zhou, F. Fang, Y.Y. Tu, J.Q. Jiang, H.X. Xu, W.L. Zhu, Acta Metall. Sin. 50, 769 (2014)

[24] H. Liu, J. Liu, P. Chen, H. Yang, Opt. Laser Technol. 118, 140 (2019)

[25] Y. Ji, W. Zhang, X. Chen, J. Li, Acta Metall. Sin. (Engl. Lett.) 29, $382(2016)$

[26] H.J. Niu, I.T.H. Chang, Microstructural evolution during laser cladding of M2 high-speed steel. Metall. Mater. Trans. A 31, 2615 (2000)

[27] C.K. Kim, J.I. Park, S. Lee, Y.C. Kim, N.J. Kim, J.S. Yang, Metall. Mater. Trans. A 36, 87 (2005) 